\title{
Formal consensus and consultation: a qualitative method for development of a guideline for dementia
}

H Trickey, I Harvey, G Wilcock, D Sharp

\begin{abstract}
Objectives-To use a formal consensus method-the nominal group techniquefor developing a clinical practice guideline to improve the diagnosis, needs assessment, and management of dementia at the primary/secondary care interface. To assess the usefulness of this method in an area where there is a dearth of clinical trials thus precluding an evidence-based approach.
\end{abstract}

Design-A qualitative and iterative method was used. The guideline was developed by an expert panel using the nominal group technique, incorporating the deliberations of a Primary Care Professionals' Task Group, recent reviews in the scientific literature, information from semistructured interviews with local specialists, and a local questionnaire survey.

Setting-Mainly Bristol but incorporating other parts of the United Kingdom especially the south and west.

Subjects-Expert panel group (including users and non-statutory organisations), local general practitioners (GPs), professionals carrying out the health check for people over 75 , local geriatricians and psychogeriatricians, and primary care professionals with an interest in dementia. Main measures-Items important in dementia from the nominal group in response to three questions on diagnosis, needs assessment, and management, ranked into a series of top 10 items for each area and allocated a score according to relative importance to each member; summary outputs from the questionnaire survey, semistructured interviews, and primary care professionals' task group.

Results-A clinical practice guideline covering diagnosis, needs assessment, and management of dementia in primary care was produced in the form of an algorithm and a management sheet.

Conclusions-A formal consensus method can be used as an acceptable alternative to the evidence-based approach when developing guidelines in situations in which evidence is scarce, in which the guideline is intended as an aid in linking different stages of care, and in which practical and political considerations-such as the links between agencies-are important. (Quality in Health Care 1998;7:192-199)

Keywords: dementia; primary care; guidelines

\section{Introduction}

GUIDELINE DEVELOPMENT

In keeping with the shift towards health services research and evidence-based health care over the past decade, development of clinical practice guidelines has moved away from small groups of experts (potentially open to bias), in favour of linking recommendations to evidence from systematic reviews of the published literature. ${ }^{1}$ Researchers who use this method hope that because recommendations derive from evidence they will be "valid" - that is, they will "lead to improvement in patient outcome at acceptable costs". ${ }^{2}$ Validity is one of the eight key attributes of a good guideline, set out by the United States Institute of Medicine, ${ }^{3}$ the others being reliability, reproducibility, clinical applicability, clinical flexibility, clarity, a multidisciplinary process, scheduled review, and documentation.

Although it would be difficult to show that guidelines which are formally linked to published evidence are more valid than those that are not (no such evaluation has been published), common sense suggests that, where feasible, incorporating available evidence is desirable. This approach has the advantage that it becomes possible to grade recommendations according to the quality of the evidence which supports them. ${ }^{4}$

However, researchers recognise that such methods come with their own problems, not least among these, the costs of time and resources involved in carrying out systematic reviews, as well as publication bias, how to incorporate qualitative evidence, ${ }^{5}$ and difficulties in translating trial results into a routine clinical setting. Additionally, there is the problem of what to do in the absence of published evidence. In practice there are always gaps and all guidelines contain a mixture of evidencelinked and consensus-based recommendations.

\section{ROLE OF PUBLISHED EVIDENCE}

Explicit links between published evidence and recommendations need not necessarily be a part of the guideline development process. Instead relevant recent reviews can be used as a basis for informing those involved in guideline development. In the field of dementia three guidelines developed with the systematic review method (two of which focused on diagnosis and initial assessment and one on the assessment of interventions in the management of behavioural and psychological aspects of dementia), and a graded review of management interventions, have recently been published. $^{6-9}$ All four reviews point to the 
dearth of good trial evidence to guide nonpharmacological management of dementia.

Of the two evidence-linked guidelines for diagnosis and initial assessment, the most recent contained only two out of 12 recommendations supported by strong research based evidence, ${ }^{6}$ one of which was the negative finding that "no single mental status test is clearly superior". The second guideline contained only one out of 17 recommendations which reflected a "high degree of clinical certainty". ${ }^{7}$ The third guideline ${ }^{9}$ had none of the nine recommendations supported by grade 1 (randomised controlled trial) evidence.

Studies that link methods of needs assessment and management to clinical outcomes of the patients with dementia and the health or wellbeing of their carer, are more difficult to find, and the links are extremely tenuous. A clinical practice guideline needs to prompt professionals to make appropriate decisions about management in the light of variable circumstances. Ways of improving such processes are more likely to be found in the experiences of professionals themselves at the present time, rather than in evaluative research.

DEMENTIA IN PRIMARY CARE

Dementia, which affects an estimated 0.7 million people in the United Kingdom, ${ }^{10}$ is an important area for development of clinical practice guidelines, especially as the prevalences available for most European countries indicate that there is a considerable problem. In, for example, Germany the prevalence of dementia rises from $7.3 \%$ at $75-79$ to $67 \%$ at $\geqslant 90$; in Italy comparable figures are $8.1 \%$ rising to $40 \%$ at $85-9$; and in Sweden, $7.9 \%$ rising to $27 \%$ at $\geqslant 90 .{ }^{11}$ This high prevalence, the anticipated demographic changes, variation in detection, ${ }^{12}$ recent availability of new drugs, ${ }^{13}$ and increasing focus on care in the community, highlight dementia as a condition with considerable importance for primary care professionals. An estimated £1039 million (1990-1 prices) was required to provide health and social services to people aged $\geqslant 65$ with Alzheimer's disease in England. ${ }^{14}$

Primary care is usually the first port of call for people with dementia and their carers, and it makes sense that guidelines be developed for use by members of the primary healthcare team. Potentially, the primary healthcare team act as gatekeepers to a web of people and organisations who provide aspects of care. However, general practitioners (GPs) often feel inadequately trained in management of dementia ${ }^{15}$ and the quality of links to specialist health, social, and voluntary services are notoriously variable.

Guidelines, for practice staff, that concentrate on detection and initial assessment of dementia, have been produced previously, ${ }^{6} 716$ whereas with exceptions, ${ }^{17}$ relatively little in the way of guidance has been published about further management. Rarely have these aspects of care been linked together in a manageable guideline format. In the United Kingdom, the Alzheimer's Disease Society (ADS) published a protocol, which covered both initial assess- ment and management of dementia. ${ }^{18}$ However, no explicit development and implementation process was used.

It has been shown that the detection of dementia can be improved through annual screening. ${ }^{19}$ However, Iliffe et al have shown that, for treatment or referral, information derived from screening will not necessarily be used to improve overall care. ${ }^{20}$ They concluded that "in the absence of agreed guidelines and resources, information derived from screening instruments may not alter clinical practice". In the light of the introduction of new drugs, the early identification of dementia is of increasing importance. The potential health gain from linking different aspects of care together in one guideline is clear. The results of existing systematic reviews indicate that a qualitative approach may be the most appropriate means of achieving this.

Quality of health care is a major item on the agenda of most policy makers, purchasers, and providers. Developing guidelines has been seen as potentially one of the most useful tools in achieving changes in behaviour and thus more uniformly high quality care. The success of a guideline can only finally be judged by whether clinical practice moves towards agreed standards of care as a result of its implementation. In dementia, there is plenty of evidence that care is not optimal ${ }^{21}$ which, together with the socioeconomic burden that results, makes it an ideal area for developing a clinical practice guideline.

The overall aim of this project was to produce a clinical practice guideline which covered detection and diagnosis of dementia, but also the wider role of primary care professionals in undertaking needs assessment, management, and review. It was clear that the scope of the guideline needed to be sufficiently broad to link vertically the different stages of care and horizontally the different professionals involved. This paper describes the pragmatic approach taken in this project to developing a clinical practice guideline in an area where few trials were available and an evidence-based approach was therefore not feasible.

\section{Method}

Our pragmatic approach to developing a guideline for use in patients with dementia at the primary-secondary care interface required various methods: a survey of local primary care professionals involved in the health check of people over 75 , interviews with local specialists and voluntary agencies, the deliberations of a primary care professionals' task group, and the views of a national expert panel using the nominal group technique.

The overall process of developing the guideline is shown in figure 1 . Broadly the resulting guideline represents the opinions of two groups, the expert panel and a primary care professionals' task group, informed by data from published reviews, interviews, and surveys. The outputs from these different processes were drawn together by the project team, which comprised a research assistant and specialists in primary care, dementia, and epidemiology and health services research. 


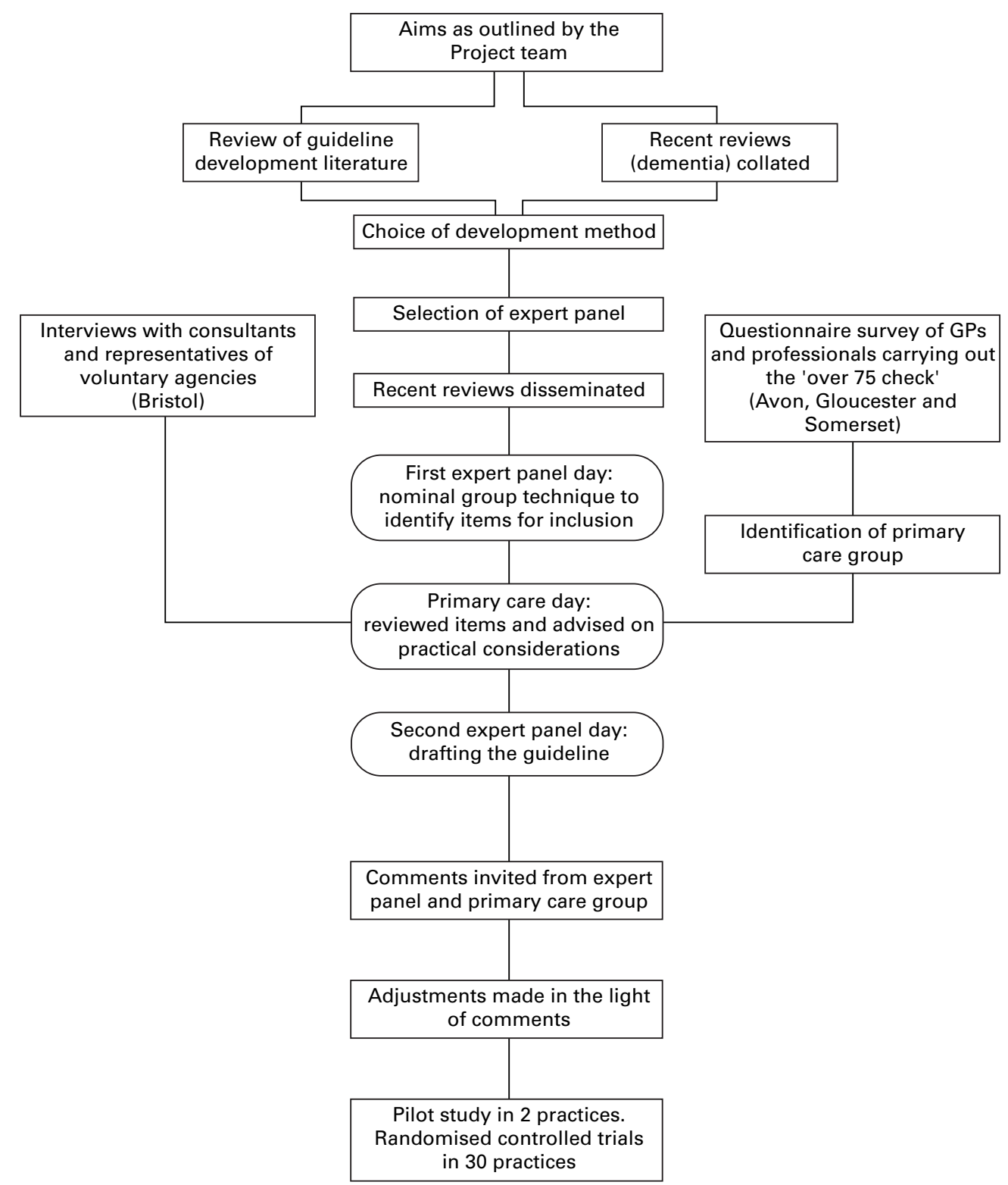

Figure 1 Dementia guideline development process. Time line $=12$ months.

LOCAL SURVEY

The purpose of the local survey was to assess professional knowledge about the treatment and management of dementia, and to identify possible barriers to implementation of a clinical guideline. Questionnaires were sent to professionals (209) carrying out the health check on people $\geqslant 75$ who were working with a one in three random sample of individual GPs in Avon, Gloucester, and Somerset Family Health Service Authorities. Respondents were asked about their current practice with a vignette describing a couple attending for a health check for people $>75$ at which it seems that the wife has signs of early dementia and the husband is having problems coping. Respondents were also asked about the perceived usefulness of guidelines, about their links with other professional and voluntary agencies, and gave information about possible barriers to use of a guideline.
INTERVIEWS WITH PROFESSIONALS AND VOLUNTARY AGENCIES

Local consultants (nine geriatricians, seven psychogeriatricians) and representatives of two voluntary agencies (Alzheimer's Disease Society and the Dementia Care Trust) were contacted by the research assistant. Semistructured interviews lasting half an hour were conducted covering links with primary care and ways in which these might be improved for patients with dementia.

NOMINAL GROUP TECHNIQUE

The nominal group technique was the formal consensus development method chosen for this study. The nominal group technique is one of several consensus techniques available ${ }^{22} 23$ the method of which has been fully described previously. ${ }^{24}$ Its strength is as a tool to generate ideas which minimises the reluctance among participants to express uncommon views and 
avoids single ideas being pursued at the expense of other important topics. It aims to avoid problems common to unstructured groups, allowing valuable discussion but avoiding domination of the decision making process by eminent or eloquent people (pertinent here as our panel contained people holding different positions in a professional hierarchy).

Before the first one day meeting, expert panel members were supplied with information about the project and with summaries of recent literature reviews. Members were asked to send in responses to three questions about diagnosis, assessment, and management of dementia (box).

PRIMARY CARE PROFESSIONALS' TASK GROUP A primary care professionals' task group was established to provide feedback and comment on the areas for inclusion chosen by the expert panel after their first meeting, and to ease implementation through exploring the practical issues involved in using guidelines for dementia in primary care, at an early stage before the mould for the guideline had been set. Also, it was intended that input from this group would balance the secondary care bias in the expert panel group and so aid implementation.
PARTICIPANTS' QUESTIONNAIRE ABOUT SATISFACTION

Participants completed a short questionnaire to evaluate the nominal group technique and to indicate whether they thought that their opinions had been represented (on a Likert scale from $1=$ fully represented to $5=$ not represented).

\section{Results}

LOCAL SURVEY

Responses from 135 (65\%) primary care professionals, mainly practice nurses, were suitable for analysis. The detailed results from this survey will be reported elsewhere. In brief, there was considerable variation in tasks that respondents thought to be within their particular remit when faced with a patient who might be presenting with dementia. Only one fifth ever used formal validated cognitive assessments as part of the health check for people over 75 . In response to the vignette, nearly $10 \%$ of nurses took no action at all and $25 \%$ simply referred the patient on. There was a lot of support for the introduction of guidelines to support nurses in their care of this group of patients.

\section{Nominal group technique as used in this project, modified from Gallagher et al (1993) ${ }^{24}$}

Expert panel-A group of 13 people with experience, expertise, and insight into dementia were selected.

Postal round-Three nominal group questions were designed and sent to each of the participating experts.

These were:

(1) What information is required in arriving at a diagnosis of dementia?

(2) What factors should be considered when assessing the needs of a person with dementia?

(3) What factors should be considered in constructing a management plan for patients with dementia?

Each person posted a list of comments and ideas in response to each of the questions to the nominal group organiser (project team research assistant).

Listing of ideas - The organiser listed all the ideas on a flip chart before the expert panel meeting.

Discussion of $i$ deas-At the expert panel meeting the organiser led a 30 minute discussion on all the ideas that had been generated in response to question 1 . This was to clarify, elaborate, defend, or dispute the items and to add new items that emerged from the discussion.

Ranking of ideas-Each member of the group chose the 10 items that they considered most important from the total list on the flip chart. They each recorded these items on a personal priority sheet.

Voting on top 10 -Each person ranked their top 10 items in order of priority, giving 10 points to the most important item and 1 point for the least important item.

Break-During a refreshment break the organiser wrote the 10 points from each of the participants alongside the items on the flip chart. Once complete, the group's top 10 items were listed on a new flip chart in order of most votes cast.

Discussion of vote- The organiser then led a short discussion about the nature and content of the top 10 items, together with feelings about items included or excluded.

Reranking and rating revised top 10 items-The organiser asked the participants to review the 10 items and to place them in a new order if they wished to do so on a personal priority sheet, giving each item a rating of between 0 and $100(0=$ low $)$. The reranking and final rating was collected by the group organiser.

The same discussion, ranking, and voting stages were repeated for questions 2 and 3. 


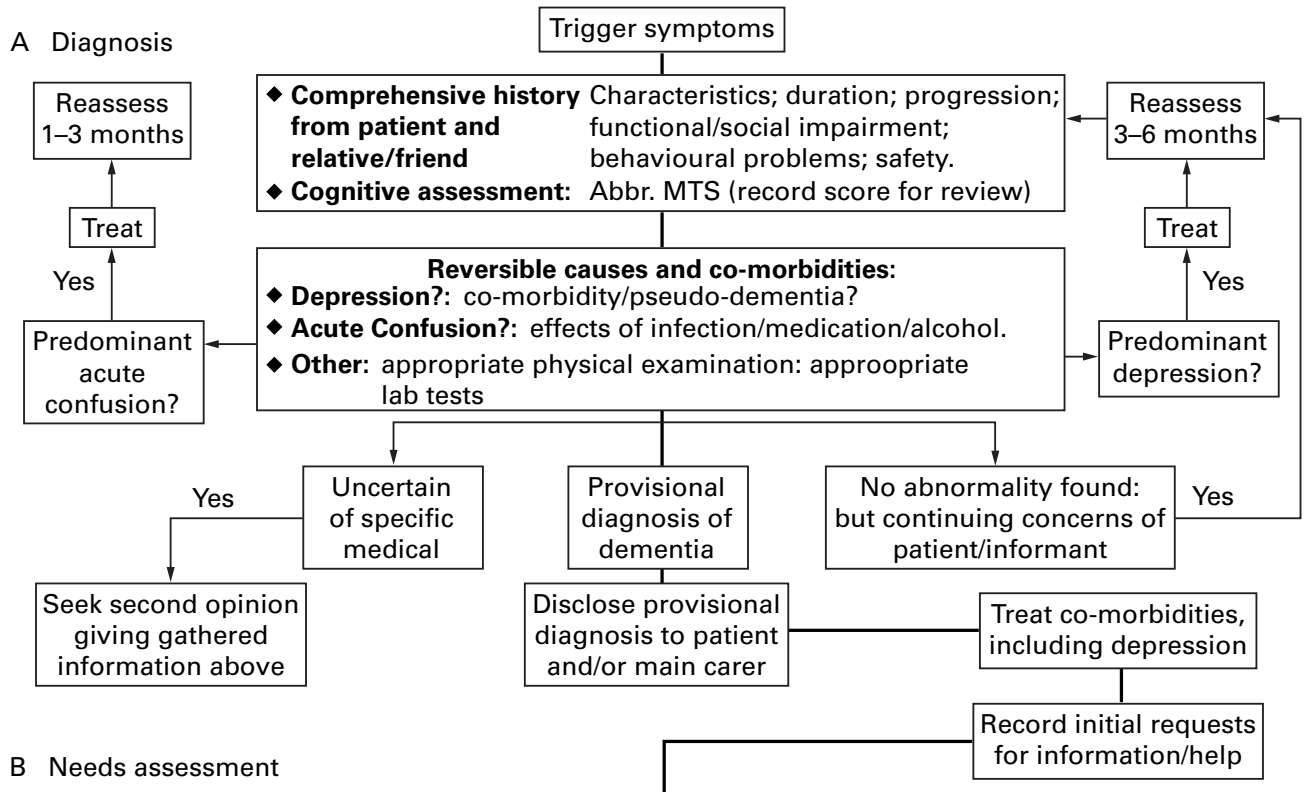

\begin{tabular}{|c|c|}
\hline $\begin{array}{l}\text { Assess and } \\
\text { record risks }\end{array}$ & $\begin{array}{l}\text { Loss of judgement: fire, falls, wandering, unsafe use of appliances } \\
\text { Driving } \\
\text { Concern regarding mistreatment of patient or carer }\end{array}$ \\
\hline $\begin{array}{l}\text { Assess and record } \\
\text { patient needs }\end{array}$ & $\begin{array}{l}\text { ADL: mobility, dressing, bathing, eating, using the toilet } \\
\text { Continence, nutrition, physical health, psychiatric symptoms } \\
\text { Adequacy of the social support network }\end{array}$ \\
\hline Carer's assessment & Offer to organise Social Services assessment \\
\hline Prompt & $\begin{array}{l}\text { Benefit entitlement? Legal advice needed? } \\
\text { Inform patient/carer of relevant local voluntary groups? }\end{array}$ \\
\hline ement & $\begin{array}{l}\text { Insert needs assessment findings into written } \\
\text { management plan kept with patient notes }\end{array}$ \\
\hline
\end{tabular}

\begin{tabular}{|c|c|}
\hline $\begin{array}{c}\text { Key person: To coordinate care: often, but not } \\
\text { always, the GP: identify to patient and main carer }\end{array}$ & $\rightarrow \begin{array}{c}\text { Name in } \\
\text { management plan }\end{array}$ \\
\hline
\end{tabular}

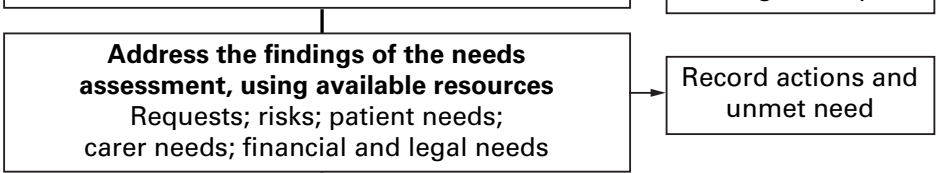

D Review carer needs; financial and legal needs

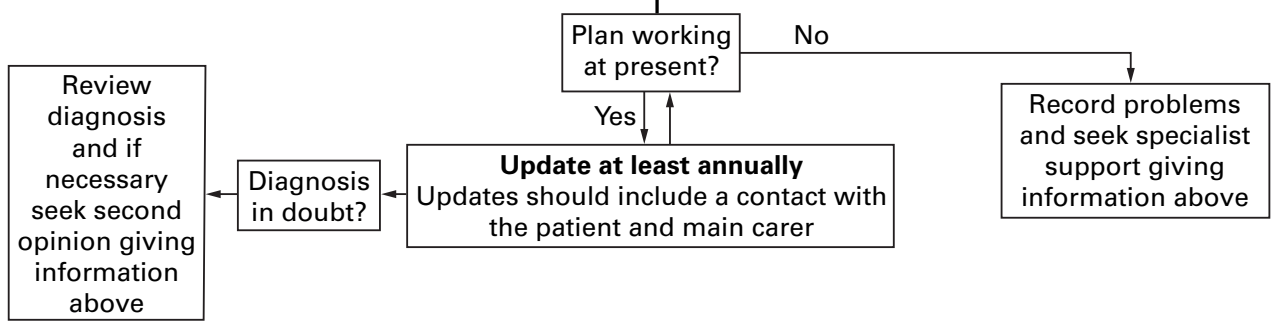

THIS GUIDELINE IS APPLICABLE FOR PERSONS LIVING BOTH IN RESIDENTIAL CARE AND IN THE COMMUNITY Dementia Guidelines Projects: 1996

Figure 2 Primary care memory change guideline.

INTERVIEWS WITH PROFESSIONALS AND VOLUNTARY AGENCIES

The interviews with this group raised three key issues: the importance of GPs carrying out tests to exclude reversible causes of the presenting symptoms to spare the patient and the NHS an unnecessary referral, the importance of a full referral letter with the main reason for referral clearly identified and the need to refer patients with a carer to ensure that the main problems are brought to light at the consultation. In particular, mention was made of the need to identify and treat depression before referral and to consider what part the primary care team might have to play in alleviating stressful home and family situations.

PRIMARY CARE PROFESSIONALS' TASK GROUP The group, consisting of seven GPs (including the GP from the expert panel, who provided a link between the groups), six health visitors, five practice nurses, and one community psychiatric nurse attached to the practice met for half a day. Participants were divided into 
Table 1 Satisfaction with nominal group technique as a guideline development tool

\begin{tabular}{|c|c|c|c|c|c|}
\hline Likert score $1-5(n=12) *$ & $\begin{array}{l}\text { How useful as a stage of } \\
\text { guideline development? }\end{array}$ & Opinions represented? & $\begin{array}{l}\text { Satisfied with items chosen } \\
\text { in the area of diagnosis? }\end{array}$ & $\begin{array}{l}\text { Satisfied with items chosen } \\
\text { in the area of assessment? }\end{array}$ & $\begin{array}{l}\text { Satisfied with items chosen in } \\
\text { the area of management? }\end{array}$ \\
\hline Median score & 2 & 2 & 2 & 2 & 2.5 \\
\hline Highest score (n) & $1(3)$ & $1(2)$ & $1(1)$ & $1(1)$ & $2(6)$ \\
\hline Lowest score (n) & $3(3)$ & $3(4)$ & $3(3)$ & $4(1)$ & $5(1)$ \\
\hline
\end{tabular}

${ }^{\star}$ Likert scale: $1=$ best possible score; $5=$ worst possible score. Participants (n) in parentheses.

three groups: (a) diagnosis, (b) assessment, and (c) management, each group being a mix of professionals and no group containing two people from the same practice. The groups were facilitated by a primary care nurse facilitator, the project team's primary care specialist, and an academic social worker.

Each group discussed the relevant list of 10 items chosen by the expert panel in terms of content, omissions, and relative importance. Practical issues such as who might use the guideline, its format, how information would be recorded and how it could be incorporated as part of the health check for people over 75, were also discussed. A report on the findings of the group was compiled. This was circulated among participants for comment, amended, and subsequently posted to the expert panel before their second meeting. The main recommendations of the group related to: the use of blood tests and other investigations to rule out potentially reversible causes of dementia, some reordering of the priorities of the diagnostic assessment items, the need to consider the patient and carer both separately and together, and the requirement to make the guideline locally feasible and flexible.

NOMINAL GROUP TECHNIQUE AND THE EXPERT PANEL

Thirteen people representing a broad base of expertise were identified through personal contacts of the project team. The resulting representation from many disciplines ensured that the guidelines were not developed by one group of professionals and subsequently imposed on another, that the roles and services provided by the various agencies were well understood, and that the links between agencies referred to in the guidelines were realistic and appropriate.

The panel included three geriatricians, two psychogeriatricians, two GPs, one community nurse, one hospital nurse, one carer, one national representative of social work managers, one Alzheimer's Disease Society representative, and one public health consultant representing a purchasing authority. Members were initially selected either as stakeholders or experts. Experts were chosen for their clinical expertise on dementia and stakeholders were chosen because of their understanding of the interests of the various professional groups. During the process it became evident that this distinction was blurred with most members holding both an expert and stakeholding role. So that the guideline could be able to be more widely generalised as well as being locally applicable the panel members were chosen to cover both national expertise and local concerns. The expert panel met twice, the first time to identify the areas for inclusion and the second time to draw together the different elements of the process into guideline format.

The first panel meeting began with a presentation which included the aims, objectives, and methods of the project. The responses to the three posted questions were then discussed, added to, and prioritised according to the nominal group technique. Non-medical panel members were relatively quiet during the discussion about diagnosis. However, all members contributed to the discussions about assessment and management. Because of the structured voting method the amount of information which each panel member contributed to choosing the final 30 items was equal (box).

At their second meeting, the expert panel was presented with the results of the local survey of primary care professionals, a digest of the material obtained through interviews with local consultants, and a summary of the report of the primary care professionals' task group. The panel then divided into three groups to develop the separate sections of the guideline (detection and diagnosis, needs assessment, and management and review), ensuring that the 30 items they had originally selected at their first meeting were included, with modifications and additions being made in the light of comments from the primary care professionals' task group. Finally, the groups came together to merge the three sections into one guideline. This was subsequently disseminated to all participants and then redrafted by the project team in the light of their comments. The final guideline was produced in two formats, as an algorithm (fig 2), and as a management sheet in line with suggestions from the primary care professionals.

\section{PARTICIPANT SATISFACTION}

Participant satisfaction, as assessed with a short questionnaire, was measured on a Likert scale (from $1=$ fully represented to $5=$ not represented); all participants scored $\leqslant 3$, most scored 2 (table). A breakdown for the three questions showed greater satisfaction with items chosen for diagnosis and assessment than those chosen for management, which might reflect the broader range of ideas generated about this last topic as well as differences in local priorities.

\section{Discussion}

This guideline development project used a multimethod approach to developing a guideline for use by primary care professionals in their face to face contacts with patients with dementia and their carers. The nominal group technique was central to the process, the members of this panel made up of experts at a national level with more local input to the 
development process being generated by questionnaires, interviews, and group meetings of local stakeholders. The total process resulted in the development of a concise guideline comprising guidance on the tasks of diagnosis, needs assessment, and management of dementia in two formats - one an A4 laminated card and the other an A5 booklet in the form of a flow chart. The format of the guidelines was the result of considerable discussion, initially at the primary care professionals' task group, (the end users), and at the second expert panel meeting. Guidelines will only bring about health gain in primary care if they are available for use at the time the patient is seen. The consensus was that simplicity and ease of use were of the utmost importance for this group of patients, who were as likely to be seen at home or in an institutional setting as in the GP's surgery. Paradoxically perhaps, the consensus approach facilitated this request and is in total contrast to the guideline developed by AHCPR for dementia in the United States ${ }^{6}$ which comprises 140 pages and would be unlikely to be implemented in United Kingdom primary care.

The particular disorder, dementia, and the broad range of issues under consideration, were, in the absence of high quality researchbased evidence, inappropriate for an evidencebased approach to guideline development. The formal consensus development method chosen, the nominal group technique, has been used in various healthcare settings to aid decision making in such situations and in particular clinical guidelines. ${ }^{25}$ However, by contrast with many other guideline development groups we used the nominal group technique in its original format as described by Delbecq and Van de $V^{2}{ }^{26}$ and did not use the Rand Corporation version which looks more like a modified Delphi technique and uses postal questionnaires to participants as part of the process. We anticipated that the formal group process laid down by the nominal group technique would allow greater participation by the non-medical members of the panel (borne out by the evenness in the satisfaction scores) and because of the breadth of the project, the separation of the generation of ideas from their evaluation and elaboration would ensure that everybody contributed to the best of their ability.

Currently, although several methods for reaching a formal consensus are available to guideline developers, informal consensus is often used where research evidence is lacking. ${ }^{27}$ Our experience of the nominal group technique suggests that this is a useful consensus tool, which facilitates an explicit decision making process. We think this method of development is likely to be successful, particularly where the guideline is primarily directed at improving processes - such as detection and good management-rather than clinical outcomes. It is useful when the emphasis is on improving the links between stages of clinical management and the links between people who are involved in the process of caring. Limiting development of guidelines to those clinical areas in which there is sufficient research-based evidence would reduce the possibility of enhancing quality of care for those other, often more difficult areas, which by their very nature do not lend themselves readily to randomised controlled trials.

Because the method of guideline development used was not entirely based on published evidence, assessment of validity cannot be made prospectively. Validity may, however, be assessed directly with a randomised controlled trial, incorporating appropriate outcome measures. Such a trial is currently being undertaken in 30 practices in the South West Region. In line with current thinking ${ }^{28}$ this randomised controlled trial uses patient and carer wellbeing as outcome measures, as well as several proxy measures including detection of dementia, detection and treatment of comorbidities (including depression and acute confusion) and referrals to other agencies. In recognition of the role of qualitative data in this complex topic area, the results of the randomised controlled trial will be augmented by semistructured interviews with primary care users and with carers.

Although the aging of the world's population can in some ways be regarded as a major achievement for medical science, the economic and political consequences provide a healthcare system such as the National Health Service with a considerable challenge. Purchasers and providers are in constant search for effective and efficient guidelines that will result in improved quality of care for patients at acceptable cost. Dementia has recently taken centre stage with the controversy over the newly licensed drugs which may have an impact early in the disease. However, as many patients will receive no benefit from them and for those that do, the benefit is likely to be short term, notable improvement in care for patients and carers at the present time is likely to result from greater attention to early diagnosis, effective needs assessment, and explicit management plans as we have described in this guideline development project.

This research was funded by the NHS South-West Research and Development Directorate and would not have been possible without the time and energy given by members of both the expert panel and the primary care professionals' group. The research was assisted by the generosity of Dr Douglas Kamerow at the Agency for Health Care Policy and Research who allowed as ancer for us an advance viewing of their dementia guideline. Special thanks are also due to Mags Watson, Jane Gilliard, and Pat Turton, ning of groups as well as the four anonymous referees who made
such helpful comments.

1 Woolf SH. Practice guidelines, a new reality in medicine. II Methods for developing guidelines. Arch Intern Med 1992;152:946-52.

2 Grimshaw J, Freemantle N, Wallace S, et al. Developing and implementing clinical practice guidelines. Quality in Health Care 1995;4:55-64.

3 Field M, Lohr K, eds, for the Committee on Clinical Practice Guidelines, Division of Health Care Services, Institute of Medicine. Guidelines for clinical practice from development to use. Washington DC: National Academy Press, 1992.

4 Mulrow C, Oxman AD, for The Cochrane Collaboration. The Cochrane library database on disc and CDRom. Oxford: The Cochrane Collaboration Update Software, 1996.

5 Jensen LA, Allen MN. Metasynthesis of qualitative findings Qualitative Health Research 1996;6:553-60.

6 US Dept of Health \& Human Services, Public Health Service, AHCPR. Recognition and Initial Assessment of Alzheimer's Disease and Related Dementia: Clinical Alzheimer's Disease and Relatice Guidelines No 191997.
Praction 
7 Report of the Quality Standards Subcommittee of the American Academy of Neurology. Practice parameters for diagnosis and

8 Melzer D, Hopkins S, Pencheon D, et al. Dementia. In: Stevens A, Raferty J, eds. Health care needs assessment: the epidemiologically based needs assessment reviews. Vol 2. Oxford: Radcliffe Medical Press, 1994:305-40.

9 Scottish Intercollegiate Guidelines. Network 22 interventions in the management of behavioural and psychological aspects of dementia. Edinburgh: Scottish Intercolkgiate Guidelines Network, February 1998.

10 Boothby H, Blizard R, Livingston G, et al. The Gospel Oak study stage III: the incidence of dementia. Psychol Med 1994;24:89-95.

11 Hofman A, Rocca W, Brayne C, et al The prevalence of dementia in Europe: a collaborative study of 1980-90 findings. Int $\mathcal{F}$ Epidemiol 1991;20:736-48.

12 O'Connor DW, Pollitt PA, Hyde JB, et al. Do general practitioners miss dementia in elderly patients? BMfournal tioners miss dement

13 Hunter R, McGill L, Bosanquet N, et al. Alzheimer's disease in the United Kingdom: developing patient and carer support strategies to encourage care in the community. Quality in Health Care 1997;6:146-52.

14 Gray A, Fenn P. Alzheimer's disease: the burden of the disease in England. Health Trends 1993;1:31-7.

15 Alzheimer's Disease Society. Right from the start: primary health care and dementia. London: Alzheimer's Disease Society, 1995.

16 Canadian Consensus Conference. Assessing dementia: the Canadian consensus. Can Med Assoc F 1991;144:851-3.

17 Jarvick LF, Wiseman E. A checklist for managing the dementia patient. Geriatrics 1991;46:31-9.
18 Haines A, Katona C. Guidelines for GPs. Management of dementia. London: Alzheimer's Disease Society, 1993.

19 Iliffe S, Haines A, Gallivan S, et al. Assessment of elderly people in general practice: 1. Social circumstances and mental state. Br f Gen Pract 1991;41:9-12.

20 Iliffe S, Mitchely S, Gould M, et al. Evaluation of the use of brief screening instruments for dementia, depression, and problem drinking among elderly people in general practice. Br f Gen Pract 1994;44:503-7.

21 Audit Commission. The coming of age: improving care services for older people. London: Audit Commission, 1998.

22 Fink A, Kosecoff J, Chassin M, et al. Consensus methods: characteristics and guidelines for use. Am $\mathcal{F}$ Public Health 1984;74:979-83.

23 Cantrill JA, Sibbald B, Buetow S. The Delphi and nominal group techniques in health services research. The International fournal of Pharmacy Practice 1996;4:67-74.

24 Gallagher $M$. The nominal group technique: a research tool for general practice?. Fam Pract 1993;10:76-81.

25 Murphy MK, Black NA, Lamping DL, et al. Consensus development methods, and their use in clinical guideline development. Health Technol Assess 1998;2.

26 Delbecq A, Van de Ven A. A group process model for problem identification and program planning. Fournal of Applied Behavioural Science 1971;7:467-92.

27 Eccles M, Clapp Z, Grimshaw J, et al. Developing valid guidelines: methodological and procedural issues from the North of England Evidence Based Guideline Development Project. Quality in Health Care 1996;5:44-50.

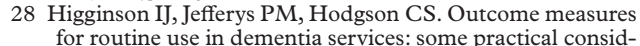
erations. Quality in Health Care 1997;6:120-3. 